\title{
Diagnostic Accuracy and Scope of Intraoperative Transoral Ultrasound and Transoral Ultrasound-Guided Fine-Needle Aspiration of Retropharyngeal Masses
}

\author{
(D).H. Vu, (D) M. Kwon, (D) S. Ahmed, (D) M. Gule-Monroe, (D) M.M. Chen, (D). Sun, (D)B.D. Fornage, (D).M. Debnam, and
}

(D) B. Edeiken-Monroe

\begin{abstract}
SUMMARY: The use of transoral sonography-guided fine-needle aspiration for intraoperative localization of retropharyngeal masses has been described by Fornage et al. The purpose of this study was to assess the accuracy of this technique. We reviewed the images and medical records of 26 patients with a retropharyngeal lesion suspicious for a metastatic lymph node of Rouviere identified on CT and/or PET/CT. There were 14 patients with a history of thyroid cancer, 7 with mucosal squamous cell carcinoma, 1 with renal cell carcinoma, 1 with parotid acinic cell cancer, 1 with metastatic colon adenocarcinoma, and 2 with no history of cancer. Intraoperative transoral sonography was performed using a commercially available endovaginal transducer. A transoral sonography-guided fine-needle aspiration was performed with a $25-\mathrm{cm}$-long 20-ga Chiba needle through a needle guide attached to the transducer shaft. Cytopathologic results were categorized as malignant, benign, or nondiagnostic. Transoral sonography and transoral sonography-guided fine-needle aspiration were performed in all patients. A diagnostic specimen was obtained in 25 of 26 (96\%) patients with a $100 \%$ overall accuracy. Twelve patients underwent subsequent transoral resection of the retropharyngeal mass. In each patient, surgical pathology confirmed the fine-needle aspiration biopsy result. In 4 patients, transoral sonography-guided injection of methylene blue was used to facilitate intraoperative localization of the metastatic retropharyngeal mass. Transoral sonography and transoral sonography-guided fine-needle aspiration of suspicious masses in the retropharyngeal space are highly accurate procedures for identification and cytologic evaluation of benign and metastatic lymph nodes of Rouviere and for presurgical localization.
\end{abstract}

ABBREVIATIONS: PTC = papillary thyroid carcinoma; $\mathrm{RCC}=$ renal cell carcinoma; $\mathrm{SCC}=$ squamous cell carcinoma; TOUS $=$ transoral ultrasound; FNA $=$ fine-needle aspiration

T he lymph nodes of Rouviere are an uncommon-but-wellknown location for regional spread of head and neck cancers. CT and, to a lesser extent, MR imaging have been the preferred techniques for identification of retropharyngeal adenopathy and for image-guided biopsy. ${ }^{1}$ A preliminary report by Fornage et $\mathrm{al}^{2}$ of direct transoral sonography (TOUS) imaging and TOUSguided fine-needle aspiration (FNA) on a small series of patients suggested the validity of the role of ultrasound in the localization, evaluation, and biopsy of retropharyngeal lesions and to facilitate transoral presurgical localization. The purpose of this report was to evaluate the accuracy of diagnostic and presurgical localization

Received April 1, 2019; accepted after revision August 12.

From the Section of Neuroradiology (T.H.V., M.K., S.A., M.G.-M., M.M.C., J.M.D., B.E.-M.) and Section of Breast Imaging (B.D.F.), Department of Diagnostic Radiology, and Department of Biostatistics (J.S.), University of Texas MD Anderson Cancer Center, Houston, Texas.

Please address correspondence to Thinh Vu, MD, Department of Diagnostic Imaging, University of Texas MD Anderson Cancer Center, 1400 Pressler St, Unit 1482, Houston, TX 77230-1402; e-mail: thinh.vu@mdanderson.org

Indicates article with supplemental on-line table.

http://dx.doi.org/10.3174/ajnr.A6236 of TOUS imaging and TOUS-FNA on suspected retropharyngeal adenopathy.

\section{MATERIALS AND METHODS}

This retrospective study, conducted between February 1, 2007 and June 30, 2016, included the images and medical records of 26 patients (10 females and 16 males) with suspicious retropharyngeal lesions detected on CT and/or on PET/CT and subsequently sampled by TOUS-FNA. The study was approved by our institutional review board and was compliant with Health Insurance Portability and Accountability Act regulations.

Summary statistics of patients and tumor characteristics were based on the frequency, mean, $\mathrm{SD}$, median, and range.

\section{TOUS Examination}

The TOUS examinations were performed in the operating room in the presence of the surgeon. The patients received general anesthesia and were intubated. The oral cavity and oropharynx were opened and stabilized with a standard McIvor-type oral retractor in preparation for placement of an endocavitary transducer. 


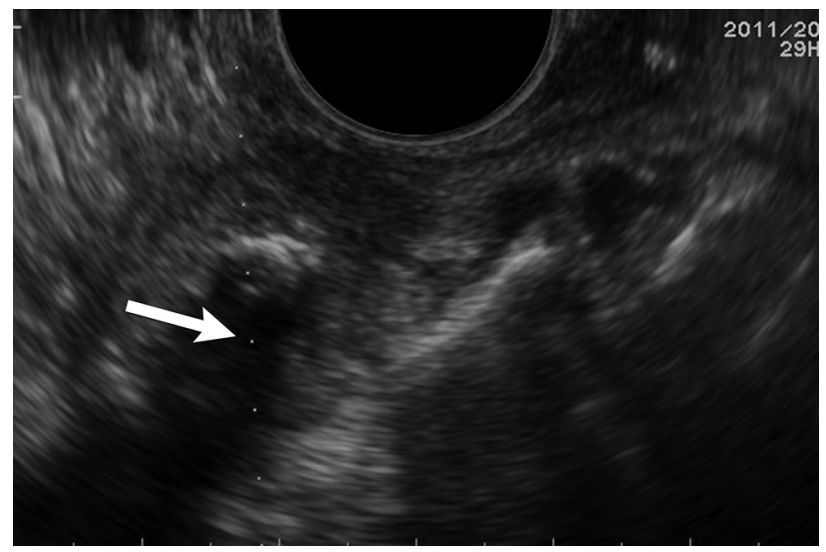

FIG 1. Biopsy line. Transverse sonography before biopsy of a partially calcified right retropharyngeal mass demonstrates a biopsy line and expected path of the biopsy needle through the lesion (white arrow).

Intraoperative TOUS imaging was performed with 1 of 2 commercially available endocavitary (endorectal or endovaginal) transducers. These included a sonography scanner (Prosound $\alpha$ 10; Hitachi-Aloka, Twinsburg, Ohio) equipped with a 3.0- to 8.5$\mathrm{MHz}$ endorectal transducer (UST-675P; Hitachi-Aloka) or a 2.5to 7.5-MHz endovaginal transducer (UST-9118; Hitachi-Aloka). Both transducers had an outer diameter of $2.3 \mathrm{~cm}$ at the tip (scan head) and offered an end-firing $180^{\circ} \mathrm{FOV}$.

Each endocavity transducer was fitted with a dedicated transducer cover (NeoGuard; CIVCO Medical Solutions, Coralville, Iowa), which was placed after a small amount of sterile coupling gel was deposited on the scan head. No coupling gel was necessary on the outside of the transducer cover because the normal pharyngeal mucosa secretions are sufficient to obtain images of diagnostic quality.

All examinations included gray-scale ultrasound and power Doppler imaging. Each lesion was measured, and when possible, measurements were performed in 2 orthogonal dimensions. Recorded ultrasound characteristics of the retropharyngeal lesions included size, echogenicity, echotexture, the presence of cystic areas, and/or calcifications and vascular flow.

Power Doppler imaging demonstrated the internal vascularity of the retropharyngeal lesions and identified the location of the internal carotid artery and the internal jugular vein.

\section{Sonography-Guided FNA Biopsy of Retropharyngeal Masses}

In preparation for a TOUS-FNA, a metallic needle guide was attached securely along the shaft of the endocavitary transducer. The needle guides included an MP-2748 for the UST-9118 endovaginal transducer or an MP-2452 for the UST- 675P endorectal transducer (Hitachi-Aloka). A 25-cm 20-ga Chiba Biopsy Needle (Cook Medical, Bloomington, Indiana) was used to accommodate the length of the needle guide.

A TOUS-FNA was performed in all patients by a neuroradiologist experienced in interventional sonography of the soft tissues of the neck. The transducer, with the needle guide attached, was inserted into the oral cavity and placed in direct contact with the pharyngeal mucosa. A built-in electronic biopsy line was

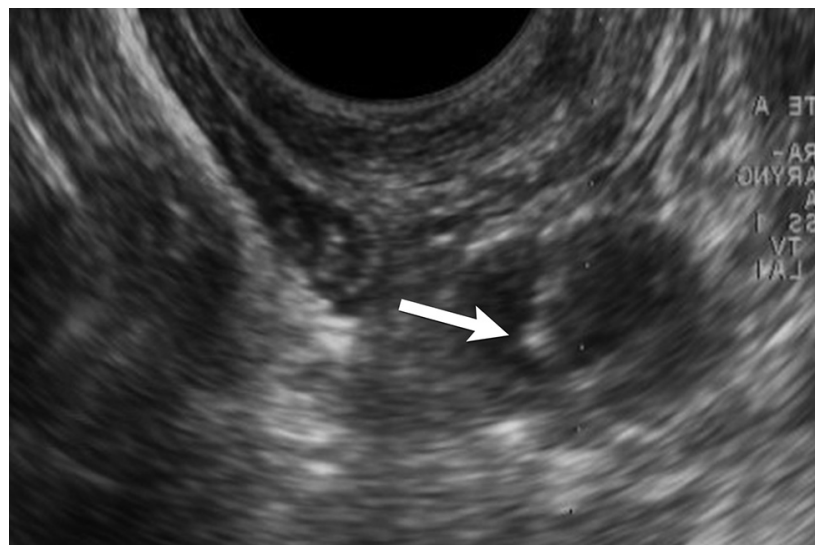

FIG 2. Needle tip visualization. Transverse sonography during biopsy of a left retropharyngeal lesion demonstrates the needle and needle tip (white arrow) accurately placed within the lesion.

activated to indicate the expected trajectory of the biopsy needle. The position of the transducer was adjusted so that the biopsy line would cross the sonographic image of the suspicious retropharyngeal mass (Fig 1).

The needle was inserted through the guide and was directed transversely through the pharyngeal wall. The needle entered the sector FOV and appeared on the sonography image as a bright echogenic line. Visualization of the needle tip was critical to allow the operator to keep the needle tip at a safe distance from the internal carotid artery and internal jugular vein as the needle was guided along the planned path to the targeted mass (Fig 2). Cellular material was aspirated from the retropharyngeal mass by applying moderate $(1-2 \mathrm{~mL})$ continuous negative pressure for approximately 10 seconds with the needle tip kept within the targeted retropharyngeal mass.

During the aspiration, the needle was moved forward and backward to mechanically tear off and aspirate sheaths of cells from the lesion and to increase the volume of the aspirate. The aspiration was completed when the suction was released. The needle-syringe assembly was withdrawn from the needle guide, and the aspirate was placed immediately and carefully on the slides to avoid crushing or drying the aspirated cells.

The slides were stained with a Romanowsky stain (Diff-Quick; Fisher Scientific, Pittsburgh, Pennsylvania) technique. A cytopathologist provided a preliminary diagnosis within approximately 30 minutes. The final diagnosis was based on the cytopathologic diagnosis and/or clinical follow-up. In 1 patient, a transoral core biopsy was performed after the TOUS-guided FNA at the request of the cytologist due to suspicion of lymphoma on the FNA aspirate and the need for a core biopsy to test for lymphoma markers. The core biopsy was performed by transoral guidance with the same apparatus used to guide the FNA.

Methylene blue $(<1 \mathrm{~mL})$ was injected with the use of TOUS guidance into the malignant lymph node found in 4 patients to facilitate intraoperative localization. The methylene blue was injected using the same needle guide through which the transoral biopsy was performed. Immediate successful transoral intraoperative resection of the metastatic adenopathy was performed without complications.

AJNR Am J Neuroradiol 40:1960-64 Nov 2019 www.ajnr.org 
Benign retropharyngeal lesions, documented by TOUS-FNA, were subsequently monitored clinically and/or by imaging.

\section{RESULTS}

The median age of the patients in this series was 53 years (range, 3-74 years). Twelve lesions ( 7 benign; 5 malignant) were located in the left retropharyngeal region, and 14 lesions ( 8 benign; 6 malignant) were in the right retropharyngeal region. The mean size of the retropharyngeal lesions was $1.6 \pm 0.8 \mathrm{~cm}$, ranging from 0.6 to $4.0 \mathrm{~cm}$ in the longest dimension, with a median size of $1.4 \mathrm{~cm}$. The mean size of the malignant retroperitoneal adenopathy was $1.6 \mathrm{~cm}$. The mean size of the benign retroperitoneal adenopathy was $1.6 \mathrm{~cm}$.

The sonographic echotexture of the lesions was 22 hypoechoic, 2 anechoic, and 2 hyperechoic. Twenty-two lesions did not exhibit normal hilar architecture, and 4 lesions had an echogenic hilum. Twenty-two lesions were solid, and 4 were cystic or had internal cystic change. Twenty-three lesions were noncalcified, and 3 contained internal calcifications. Doppler examination of the lesions showed 18 lesions without disorganized flow and 8 lesions with disorganized flow.

The 26 retropharyngeal lesions were detected by CT $(n=$ $20)$ and/or PET/CT $(n=6)$. A TOUS-guided biopsy of the suspicious retropharyngeal lesions was performed in each of the 26 patients with the needle accurately positioned under sonographic guidance within the retropharyngeal lesion. All TOUSFNAs were well-tolerated. There were no immediate or delayed complications during or after the biopsies.

Twenty-five of 26 TOUS-FNA aspirates were diagnostic. One TOUS-FNA was nondiagnostic, and 1 TOUS-FNA was inadequate to exclude lymphoma. In the patient in whom aspiration was inadequate to exclude lymphoma, a TOUS core biopsy was performed, which revealed reactive hyperplasia of the lymph node but no evidence of lymphoma. In the 1 patient with nondiagnostic TOUS-FNA, direct smears demonstrated a few benign mucosal epithelial cells and rare connective tissue elements. No evidence of lymph node sampling was present. Follow-up MR imaging showed that the node remained stable but prominent in size $(1.0 \mathrm{~cm})$. The patient died of recurrent squamous cell carcinoma (SCC) in the maxillary sinus and, therefore, was lost to follow-up.

The final diagnosis of the TOUS-FNA was consistent with the preliminary diagnosis in all 25 patients who underwent diagnostic aspirations. Surgical pathology results were obtained in 10 (38\%) of the 26 patients. In the $16(62 \%)$ patients who did not undergo surgical intervention, clinical or imaging follow-up supported the final diagnosis. This included the 1 patient who underwent TOUS-FNA in a lymph node detected on PET/CT that subsequently regressed on follow-up evaluation. One of the 26 patients was lost to follow-up after TOUS-FNA and core biopsy revealed a reactive lymph node on cytology.

Results from the TOUS-FNAs are summarized in the On-line Table.

\section{Patient Population}

The primary diseases in the 26 patients included papillary thyroid carcinoma (PTC) $(n=14)$, mucosal SCC $(n=7)$, parotid acinic cell carcinoma $(n=1)$, metastatic colorectal cancer $(n=1)$, renal cell carcinoma (RCC) $(n=1)$, and no cancer history in 2 patients.

Benign retropharyngeal lesions were documented in 14 patients. These included patients with PTC $(n=6)$, mucosal SCC $(n=4)$, parotid acinic cell carcinoma $(n=1)$, metastatic colorectal cancer $(n=1)$, and no history of malignancy $(n=2)$. In all the cases in which cytology was negative for PTC, thyroglobulin assays were not performed on the aspirates once we had an adequate cellular sample. Among patients with benign TOUS-FNAs, the retropharyngeal lesions of interest included the following:

- One patient with PTC whose initial cytology was of concern for reactive adenopathy versus lymphoma. A TOUS core biopsy confirmed benign reactive hyperplasia with no evidence of lymphoma.

- One patient with no history of malignancy, whose retropharyngeal mass was incidentally visualized on CT performed at another facility for dysphagia. The TOUS-FNA diagnosed a ganglion cyst that was resected transorally.

- A second patient with no previous history of malignancy was a 3-year-old male child with a calcified right retropharyngeal mass found incidentally on a neck CT. A TOUS-FNA revealed dense acellular calcific debris. The lymph node was resected, and the histology was concordant with the initial cytologic finding with no tumor present.

- One patient had a base of tongue SCC; the patient's left retropharyngeal/parapharyngeal space mass was FDG-avid on staging PET/CT. TOUS-FNA revealed a salivary gland neoplasm with cystic degeneration. This was surgically resected, and the final histology was consistent with basal cell adenoma of salivary gland origin.

Metastatic retropharyngeal adenopathy was documented in 11 patients. These included patients with PTC $(n=8)$, mucosal SCC $(n=2)$, and metastatic adenopathy from undiagnosed PTC in the patient with RCC $(n=1)$. Among patients with metastatic TOUS-FNAs, the retropharyngeal masses of interest included the following:

- One patient with RCC in whom TOUS-FNA revealed a malignant lymph node from an undiagnosed PTC.

- One patient with PTC whose metastatic adenopathy included a cystic lymph node. The lymph node metastasis was diagnosed by elevated thyroglobulin assay on the aspirate.

One patient with a history of recurrent facial SCC had a nondiagnostic TOUS-FNA of a prominent $1.0-\mathrm{cm}$ right lateral retropharyngeal node detected on PET/CT. Follow-up MR imaging showed that the lymph node remained stable but prominent in size $(1.0 \mathrm{~cm})$. The patient experienced recurrent SCC in the maxillary sinus and died of this disease within 2 years of the documented retropharyngeal metastatic adenopathy (and was thus lost to follow-up).

\section{Illustrative Cases}

True-Positive Cytology.

- A right $3.0 \times 2.0 \times 1.5 \mathrm{~cm}$ retropharyngeal lymph node of concern was detected on staging $\mathrm{CT}$ in a 65 -year-old man 

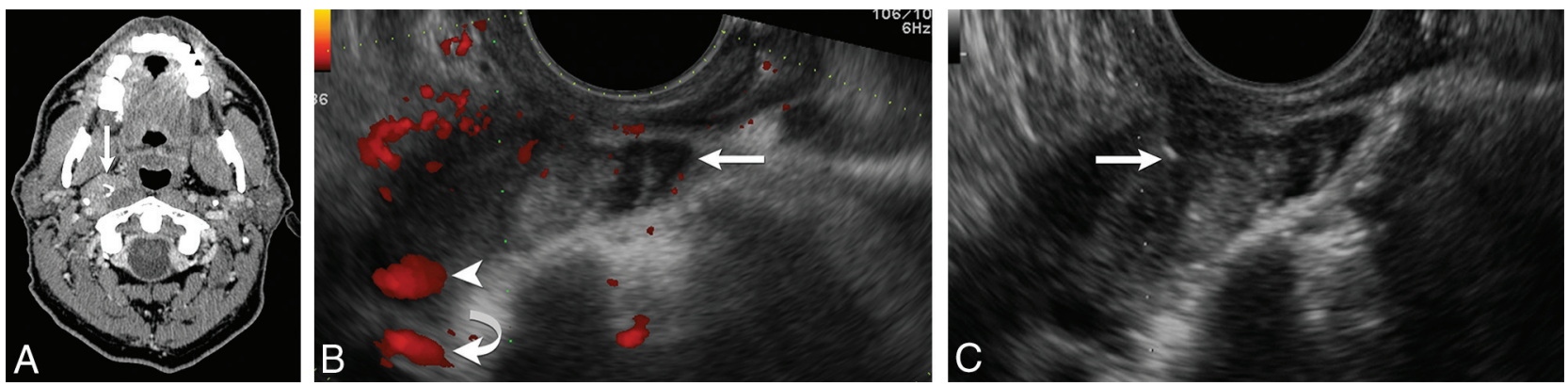

FIG 3. True-positive cytology. A 65-year-old man with a history of papillary thyroid cancer and a right retropharyngeal mass. $A$, Axial contrastenhanced CT scan shows an enlarged right retropharyngeal node (arrow) with internal calcification. B, Transverse power Doppler sonography shows the lesion (arrow) and its relationship to the internal carotid artery (arrowhead) and internal jugular vein (curved arrow). C, Transverse sonography during biopsy of the mass documents placement of needle tip (white arrow) within the lesion before and during aspiration. Cytology revealed metastatic papillary thyroid cancer.
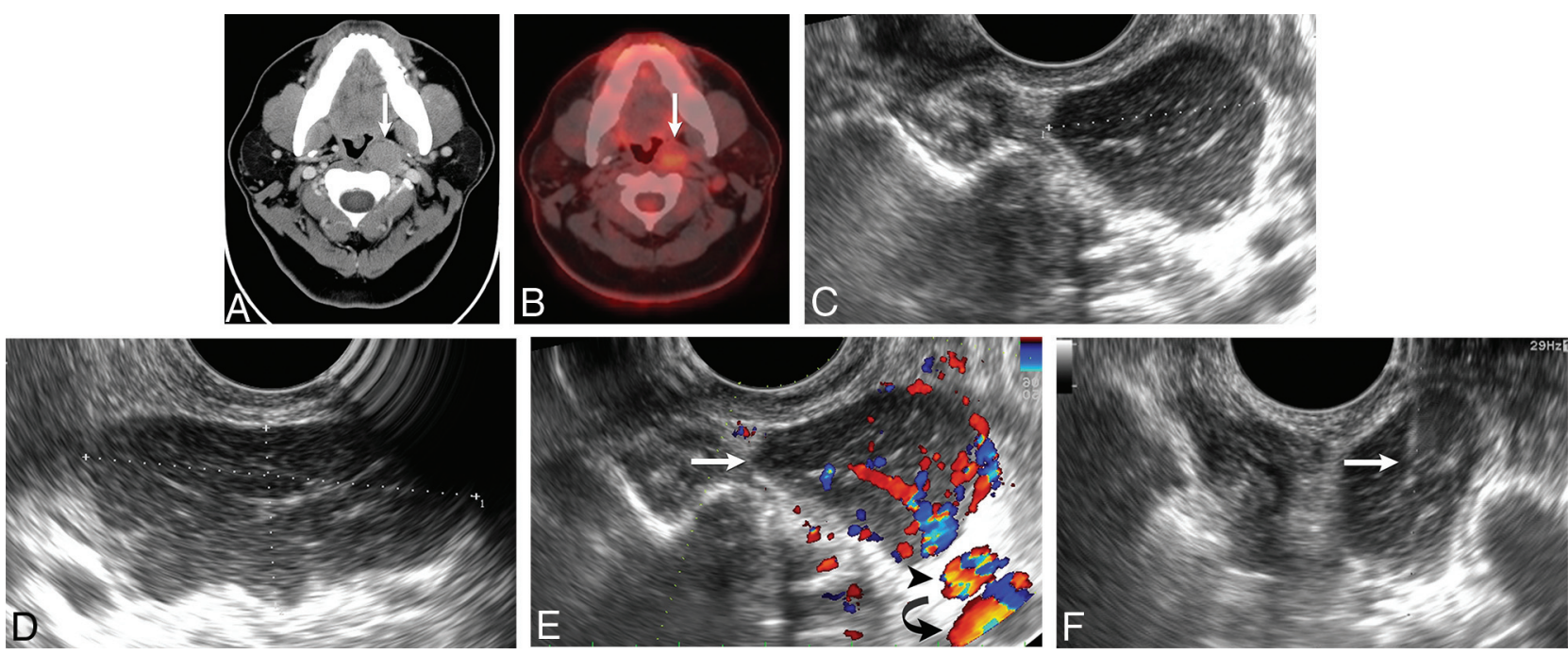

FIG 4. True-negative cytology. A 38-year-old woman with a history of papillary thyroid cancer and left retropharyngeal mass. A, Axial contrast-enhanced CT scan shows an enlarged left retropharyngeal node (arrow). B, Axial PET/CT shows that the enlarged left retropharyngeal node has mild FDG avidity (arrow). Transverse $(C)$ and sagittal oblique $(D)$ sonography reveals an enlarged hypoechoic left lateral retropharyngeal node measuring $2.5 \times 4.3 \times 1.9 \mathrm{~cm}$. E. Transverse color Doppler sonography shows the vascular flow of the lesion (white arrow) and its relationship to the internal carotid artery (black arrowhead) and internal jugular vein (black curved arrow). F, Transverse sonography during biopsy of the mass documents placement of needle tip (arrow) within the lesion before and during aspiration. Cytology revealed benign reactive hyperplasia.

with a history of PTC. A TOUS-FNA was performed, and cytology showed metastatic PTC. The patient subsequently underwent transoral robotic resection of the lesion aided by localization with methylene blue inserted by TOUS guidance. Histology confirmed the cytologic diagnosis of metastatic PTC (Fig 3).

\section{True-Negative Cytology.}

- A large $4.3 \times 2.5 \times 1.9 \mathrm{~cm}$ left lateral retropharyngeal mass was found on restaging CT and PET/CT in a 38-year-old woman with a history of PTC. TOUS-FNA was performed, and cytology revealed benign reactive hyperplasia. There was clinical concern for lymphoma; therefore, a core biopsy was performed. The diagnosis of lymphoma was excluded, and the diagnosis was confirmed as a benign reactive lymph node (Fig 4).

\section{DISCUSSION}

The retropharyngeal space is a known area of local regional metastasis of head and neck cancers. It is defined as a potential midline space located between the pharyngeal constrictor muscle anteriorly and prevertebral muscles posteriorly. It is bordered by the pharyngeal mucosal space anteriorly, the carotid space laterally, and the prevertebral portion of the perivertebral space posteriorly. It communicates with the parapharyngeal space and contains adipose tissue and lymph nodes known as the nodes of Rouviere.

The success of the TOUS route to evaluate the retropharyngeal space previously reported included a small number of patients. ${ }^{2}$ Our study included an expanded number of patients to evaluate the reliability and accuracy of TOUS and of the TOUSguided biopsy technique. Surgical pathology or imaging followup or both were used as the standard of reference. 
Imaging techniques used to identify, evaluate, and localize the lymph nodes of Rouviere included CT and MR imaging using the anterior and retromandibular approach and sonography using the transoral approach. Standard 2D ultrasound of the retropharyngeal region is limited by the overlying osseous structures. The advantages of TOUS include evaluation of the retropharyngeal lesion in the transverse and sagittal planes; identification of sonographic characteristics suggestive of papillary thyroid cancer such as intranodal vascularity, calcification, and cystic change; definition of the relationship between the retropharyngeal lesion and the internal carotid artery and internal jugular vein; and graded compression of the pharyngeal wall with the transducer to assess the presence or absence of a fat plane between the lesion and the carotid artery, which provides critical information for the operating surgeon.

The TOUS imaging and biopsy technique permits a pathologic diagnosis of retropharyngeal adenopathy and facilitates minimally invasive transoral resection. This system provides a reasonable replacement for the more challenging CT-guided needle biopsy. The needle guide attached to the endocavitary sonography transducer defines the expected trajectory of the biopsy needle and facilitates placement of the biopsy needle tip within the targeted lesion.

Two of the retropharyngeal lymph nodes that underwent TOUS-FNA were of particular interest and demonstrated the usefulness of this procedure in differing clinical scenarios. One was in a patient with PTC in whom the preliminary cytology of the TOUS-FNA suggested a reactive lymph node versus concern for lymphoma. A core biopsy was required to evaluate lymphoma. The TOUS-guided core biopsy excluded lymphoma and confirmed the diagnosis of benign reactive lymphoid hyperplasia obtained on TOUS-FNA. The second patient had RCC, and TOUS-FNA showed that the retropharyngeal adenopathy was due to unsuspected undiagnosed PTC.

Accurate preoperative imaging is required to assess the need for surgical intervention and surgical guidance. TOUS allows aspiration of the retropharyngeal mass to distinguish benign from malignant disease. TOUS assists in the surgical approach by demonstrating the relationship between a retropharyngeal lesion and the adjacent vasculature to guide the surgeon.

More than half of the patients in our series $(n=14)$ who underwent TOUS-FNA of the retropharyngeal nodes had a history of PTC. The importance of this procedure is to determine the status of the neck nodes, which is an important prognostic factor in patients with well-differentiated thyroid cancer. ${ }^{3,4}$ In addition, aggressive surgical resection of the retropharyngeal metastasis is the preferred treatment because it correlates with a decreased cancer-specific mortality rate and improved disease-free survival. Surgical intervention was facilitated in our series by injection of methylene blue through the TOUS-FNA apparatus at the time of biopsy-proved metastatic adenopathy.

In our experience, evaluation of retropharyngeal adenopathy with TOUS-FNA yields an accurate cytologic evaluation (100\%) and diagnostic yield (96\%) without complications. Our diagnostic yield was higher than that achieved in previously reported series in which evaluation and biopsy of a retropharyngeal or parapharyngeal mass were performed by CT or MR imaging either from a retromandibular or paramaxillary approach without conscious sedation or general anesthesia. Maghami et $\mathrm{al}^{5}$ and Lai et $\mathrm{al}^{6}$ used MR imaging to guide the diagnostic biopsy of retropharyngeal masses in $78 \%$ and $71 \%$ of patients, respectively. Sherman et $\mathrm{al}^{7}$ reported a diagnostic accuracy of $85 \%$ in 27 patients using CT to guide the biopsy of parapharyngeal space lesions. Our high diagnostic yield and accuracy rates are likely due to our FNA technique. A needle guide attached to the probe assisted in alignment of the needle with the probe scan plane and allowed visualization of the trajectory of the needle tip as it is moved toward the target during the procedure.

A limitation of this study is that all TOUS and TOUS-FNA procedures were performed in the operative theater with the patient under general anesthesia, which may add increased risk to the patient. In addition, this procedure involves the assistance of a surgeon to open the oral cavity to allow adequate exposure to the posterior oropharyngeal wall for sonographic scanning and biopsy. However, these limitations may be negated because the intraoral approach, in the operating room in the presence of the surgeon, allows immediate intraoral surgical excision rather than the more challenging extraoral transcervical approach.

\section{CONCLUSIONS}

A TOUS-guided biopsy is a safe, feasible, accurate, and reliable diagnostic procedure to differentiate benign from malignant retropharyngeal adenopathy and provides guidance for minimally invasive transoral resection.

\section{ACKNOWLEDGMENTS}

The authors thank our Department of Scientific Publications at the University of Texas MD Anderson Cancer Center, Houston, Texas, for their kind assistance in editing.

\section{REFERENCES}

1. Connor SE, Chaudhary N. CT-guided percutaneous core biopsy of deep face and skull-base lesions. Clin Radiol 2008;63:986-94 CrossRef Medline

2. Fornage BD, Edeiken BS, Clayman GL. Use of transoral sonography with an endocavitary transducer in diagnosis, fine-needle aspiration biopsy, and intraoperative localization of retropharyngeal masses. AJR Am J Roentgenol 2014;202:W481-86 CrossRef Medline

3. Mazzaferri EL, Jhiang SM. Long-term impact of initial surgical and medical therapy on papillary and follicular thyroid cancer. $A m J$ Med 1994;97:418-28 CrossRef Medline

4. Stojadinovic A, Shoup M, Nissan A, et al. Recurrent differentiated thyroid carcinoma: biological implications of age, method of detection, and site and extent of recurrence. Ann Surg Oncol 2002;9:78998 CrossRef Medline

5. Maghami EG, Bonyadlou S, Larian B, et al. Magnetic resonance imaging-guided fine-needle aspiration biopsies of retropharyngeal lesions. Laryngoscope 2001;111:2218-24 CrossRef Medline

6. Lai A, Maghami E, Borges A, et al. MRI-guided access to the retropharynx. J Magn Reson Imaging 2003;17:317-22 CrossRef Medline

7. Sherman PM, Yousem DM, Loevner LA. CT-guided aspirations in the head and neck: assessment of the first 216 cases. AJNR Am J Neuroradiol 2004;25:1603-07 Medline 